\title{
A Survey of Faculty Development Practices
}

\section{Glenn Erickson}

Univesity of Rhode Island

The POD Network decided to conduct a survey of faculty development practices for several reasons. First, many people believed that the "faculty development movement" was a casualty of tight budgets and an inevitable loss of faith in yesterday's strategies; some of us had our doubts. Also, we were curious about just what kinds of services were being offered by whom in our colleges and universities and whether things had changed dramatically in the years since Centra's survey (Centra, 1976). Finally, we had never had a decent mailing list of "development" practitioners, yet we claimed to be committed to putting such folks in touch with each other.

In mid-1984, we mailed a letter to the chief academic officers of the 1,588 four-year (or more) colleges and universities in the U.S. asking them to send us the names and addresses of people at their institutions who directed or coordinated ". . . what are typically called faculty development, instructional development, or teaching improvement programs or activities." About 1,200 responded to that letter or to a subsequent prompt; 450 indicated that they didn't have any formal program; the remaining 750 sent us the names of nearly 1000 coordinators, directors, committee chairs, or administrators.

We asked those 1,000 people to complete a 47 -item questionnaire adapted from Centra's (1976) earlier survey. The questionnaire listed 40 different activities in five categories: 
workshops and seminars; assessment practices; individual consultation; grants, leaves, and exchanges; and other practices. (Table 2 lists the 40 activities.) We asked people to check those activities that had been generally available to their faculties within the most recent $12-18$ months. The questionnaire also asked who was responsible for planning and coordinating such services, how their current investment in faculty development compared to that of three years ago, and for some demographic information. Unlike Centra's survey, POD's did not ask for estimates of the extent of participation or degree of effectiveness.

We received about 800 completed questionnaires from 650 different institutions after mailings in the spring and fall of 1985. We combined multiple returns from individual institutions, discarded incomplete questionnaires, and excluded the few from two-year colleges. We then had 630 usable responses, a coordinator response rate of about 79\%. Table 1 describes the sample of respondents by region, institutional type, enrollment, and whether public or private. We have completed questionnaires from half of the four-year colleges, universities, and professional schools in the Southeast, Midwest, and West. Perhaps because of a high proportion of very small schools in the Northeast, we have responses from only $38 \%$ of the schools in that region.

The responses to survey questions about responsibility for and relative investment in services suggest that the "faculty development movement" is neither dead nor even in decline. Ten years ago, $41 \%$ of the 408 four-year colleges and universities responding to Centra's survey claimed " . . . an on-campus person or unit(s) for faculty development or instructional improvement . . ." (1976, p. 34); 44\% claimed such a person or unit in 1985 . Moreover, 66\% of the 1985 respondents indicated that their institutions' current investment in faculty, instructional, and professional development was much or somewhat greater than it had been three years earlier; only $9 \%$ rated their relative investment as somewhat or much less now. Although we can all name centers and programs that have been shut down or dramatically scaled back, responses to the POD survey suggest that more programs are starting up than closing down. We can't know, of course, what the survival rate will be for current programs (half were created since 1981), but we have no evidence here to suggest faculty development is dying. 
TABLE 1

Survey Questionnaire Respondents by Region. Enrollment, Public vs. Private. and Institutional Type

\begin{tabular}{|c|c|c|c|c|c|c|c|c|c|c|c|c|c|c|c|}
\hline $\begin{array}{c}\text { Region and } \\
\text { institutional } \\
\text { type }\end{array}$ & $\begin{array}{l}\text { Un } \\
\text { pub́ }\end{array}$ & $\begin{array}{l}\text { ider } \\
\text { ooo } \\
\text { prv }\end{array}$ & $\begin{array}{r}1: \\
2: 5\end{array}$ & $\begin{array}{l}000- \\
500 \\
\text { prv }\end{array}$ & $\begin{array}{r}2: 5 \\
5: 9 \\
\text { pub }\end{array}$ & $\begin{array}{l}500- \\
000 \\
\text { prv }\end{array}$ & $\begin{array}{l}5.00 \\
10.0 \\
\text { pub }\end{array}$ & $\begin{array}{l}\text { po- } \\
\text { prve }\end{array}$ & $\begin{array}{l}10.0 \\
20: 0 \\
\text { pub }\end{array}$ & $\begin{array}{l}\text { OO- } \\
\text { o0 } \\
\text { prv }\end{array}$ & $\begin{array}{l}\text { Ove } \\
20,0 \\
\text { pub }\end{array}$ & $\begin{array}{l}\text { r } \\
\text { porv } \\
\text { prv }\end{array}$ & pub & $\begin{array}{l}\text { All } \\
\text { Pru }\end{array}$ & a 1 \\
\hline $\begin{array}{l}\text { Northeast } \\
\text { Four-year } \\
\text { University } \\
\text { Professional }\end{array}$ & $\begin{array}{l}2 \\
0 \\
2\end{array}$ & $\begin{array}{r}31 \\
\mathbf{0} \\
7\end{array}$ & $\begin{array}{l}\mathbf{5} \\
\mathbf{0} \\
\mathbf{0}\end{array}$ & $\begin{array}{r}44 \\
3 \\
2\end{array}$ & $\begin{array}{r}10 \\
0 \\
0\end{array}$ & $\begin{array}{r}10 \\
4 \\
0\end{array}$ & $\begin{array}{l}9 \\
2 \\
0\end{array}$ & $\begin{array}{l}2 \\
5 \\
0\end{array}$ & $\begin{array}{l}5 \\
6 \\
0\end{array}$ & $\begin{array}{l}1 \\
2 \\
0\end{array}$ & $\begin{array}{l}\mathbf{0} \\
\mathbf{3} \\
\mathbf{0}\end{array}$ & $\begin{array}{l}0 \\
2 \\
0\end{array}$ & $\begin{array}{l}31 \\
11 \\
2\end{array}$ & $\begin{array}{r}88 \\
16 \\
9\end{array}$ & $\begin{array}{r}119 \\
27\end{array}$ \\
\hline a $11 \mathrm{NE}$ & 4 & 38 & 5 & 49 & 10 & 14 & 11 & 7 & 11 & 3 & 3 & 2 & 44 & 113 & \\
\hline $\begin{array}{l}\text { Southeast } \\
\text { Four-year } \\
\text { University } \\
\text { Professional }\end{array}$ & $\begin{array}{l}2 \\
0 \\
2\end{array}$ & $\begin{array}{r}38 \\
0 \\
5\end{array}$ & $\begin{array}{l}8 \\
1 \\
2\end{array}$ & $\begin{array}{r}33 \\
\mathbf{0} \\
1\end{array}$ & $\begin{array}{r}15 \\
4 \\
1\end{array}$ & $\begin{array}{l}2 \\
1 \\
0\end{array}$ & $\begin{array}{l}8 \\
5 \\
0\end{array}$ & $\begin{array}{l}1 \\
1 \\
0\end{array}$ & $\begin{array}{r}4 \\
1\end{array}$ & $\begin{array}{l}\mathbf{1} \\
\mathbf{0} \\
\mathbf{0}\end{array}$ & $\begin{array}{l}0 \\
7 \\
0\end{array}$ & $\begin{array}{l}\mathbf{0} \\
\mathbf{0} \\
\mathbf{0}\end{array}$ & $\begin{array}{r}37 \\
28 \\
6\end{array}$ & $\begin{array}{r}75 \\
2 \\
6\end{array}$ & $\begin{array}{r}112 \\
30 \\
12\end{array}$ \\
\hline all SE & 4 & 43 & 11 & 34 & 20 & 3 & 13 & 2 & 16 & 1 & 7 & $\mathbf{o}$ & 71 & 83 & \\
\hline $\begin{array}{l}\text { Midwest } \\
\text { Four-year } \\
\text { University } \\
\text { Professional }\end{array}$ & $\begin{array}{l}2 \\
0 \\
1\end{array}$ & $\begin{array}{r}64 \\
22 \\
11\end{array}$ & $\begin{array}{l}6 \\
1 \\
1\end{array}$ & $\begin{array}{r}48 \\
0 \\
2\end{array}$ & $\begin{array}{r}10 \\
0 \\
0\end{array}$ & $\begin{array}{l}7 \\
1 \\
0\end{array}$ & $\begin{array}{r}10 \\
4 \\
0\end{array}$ & $\begin{array}{l}1 \\
5 \\
0\end{array}$ & $\begin{array}{r}5 \\
11 \\
0\end{array}$ & $\begin{array}{l}\mathbf{0} \\
\mathbf{2} \\
\mathbf{0}\end{array}$ & $\begin{array}{r}0 \\
11 \\
0\end{array}$ & $\begin{array}{l}\mathbf{0} \\
\mathbf{0} \\
\mathbf{0}\end{array}$ & $\begin{array}{r}33 \\
27 \\
2\end{array}$ & $\begin{array}{r}120 \\
10 \\
13\end{array}$ & $\begin{array}{r}153 \\
37 \\
15\end{array}$ \\
\hline a $11 \mathrm{MW}$ & 3 & 77 & 8 & 50 & 10 & 8 & 14 & 6 & 16 & 2 & 11 & $\mathbf{o}$ & 62 & 143 & \\
\hline $\begin{array}{l}\text { West } \\
\text { Four-year } \\
\text { University } \\
\text { Professional }\end{array}$ & $\begin{array}{l}1 \\
0 \\
0\end{array}$ & $\begin{array}{r}18 \\
2 \\
7\end{array}$ & $\begin{array}{l}\mathbf{5} \\
\mathbf{0} \\
\mathbf{0}\end{array}$ & $\begin{array}{r}19 \\
1 \\
1\end{array}$ & $\begin{array}{l}8 \\
3 \\
0\end{array}$ & $\begin{array}{l}3 \\
1 \\
0\end{array}$ & $\begin{array}{l}5 \\
5 \\
1\end{array}$ & $\begin{array}{l}0 \\
2 \\
0\end{array}$ & $\begin{array}{r}6 \\
11 \\
0\end{array}$ & $\begin{array}{l}0 \\
2 \\
0\end{array}$ & $1 \frac{1}{1}$ & $\begin{array}{l}0 \\
1 \\
0\end{array}$ & $\begin{array}{r}26 \\
30 \\
1\end{array}$ & $\begin{array}{r}40 \\
9 \\
8\end{array}$ & $\begin{array}{r}66 \\
39 \\
9\end{array}$ \\
\hline a11 w & 1 & 27 & 5 & 21 & 11 & 4 & 11 & 2 & 17 & 2 & 12 & 1 & 57 & 57 & \\
\hline $\begin{array}{l}\text { A11 } \\
\text { Four-year } \\
\text { University } \\
\text { Professional }\end{array}$ & $\begin{array}{l}7 \\
0 \\
5\end{array}$ & $\begin{array}{r}151 \\
30\end{array}$ & $\begin{array}{r}24 \\
2 \\
3\end{array}$ & $\begin{array}{r}144 \\
4 \\
6\end{array}$ & $\begin{array}{r}43 \\
7 \\
1\end{array}$ & $\begin{array}{r}22 \\
7 \\
0\end{array}$ & $\begin{array}{r}32 \\
16 \\
1\end{array}$ & $\begin{array}{r}4 \\
13 \\
0\end{array}$ & $\begin{array}{r}20 \\
39 \\
1\end{array}$ & $\begin{array}{l}2 \\
6 \\
0\end{array}$ & $\begin{array}{r}32 \\
0\end{array}$ & $\begin{array}{l}\mathbf{0} \\
\mathbf{3} \\
\mathbf{0}\end{array}$ & $\begin{array}{r}127 \\
96 \\
11\end{array}$ & $\begin{array}{r}323 \\
37 \\
36\end{array}$ & $\begin{array}{r}450 \\
133 \\
47\end{array}$ \\
\hline combined & 12 & 185 & 29 & 154 & 51 & 29 & 49 & 17 & 60 & 8 & 33 & 3 & 234 & 396 & 630 \\
\hline
\end{tabular}

Note: Northeast includes CT, DC, DE, MA, MD, ME, NH, NJ, NY, PA, RI, VT; SOUtheast--AL, AR: FL: GA: KY: LA: MS, NC, PR, SC, TN, 
Tables 2-8 provide information about the activities offered to faculty in responding institutions. Table 2 reports the percentages of institutions that offered each of the services or activities within the preceding 18 months. It shows, for example, that student ratings of instruction were available on more than 95\% of these campuses, although under half provided individual help from trained consultants in interpreting such ratings. Tables 3-8 report the median number of faculty development practices in each category that institutions made available to their faculties. The tables also show how many institutions offered half or more of the opitons listed in the questionnaire. Institutions are sorted by geographic region, type, and enrollment in the tables.

It's apparent that the most popular category of practices is the traditional one of grants, awards, leaves, and exchanges (Table 6). Individual consultation services (Table 5) are available at the fewest number of institutions. Regional differences are not dramatic, but there seems to be more variety in services as one moves west (Tables 3-8). Not surprisingly, larger institutions - especially universities-offer a greater variety of services than smaller ones. Tables 2-8 present the findings in considerable detail because information about faculty development practices in particular kinds of settings is likely to be of more interest than sweeping generalizations.

We were also curious about who was responsible for coordinating faculty development practices on these campuses. There were faculty development committees at $62 \%$ of the schools (also true in 1976), but they apparently serve advisory roles. Committees actually coordinated or provided services in only $14 \%$ of the institutions. Committees shared reponsibility with an administrator at $4 \%$ of the schools. Fourteen percent of the institutions had centers or programs, and another $14 \%$ had coordinators or directors of faculty development. Most typically, however, a dean or other administrator had responsibility for faculty development as one of his or her several duties. Predictably, institutions with a center or designated coordinator offered the most services; fewest services were available on campuses where faculty development was one among many of an adminstrator's responsibilities. 


\section{TABLE 2}

Percentages of Private and Public Four-year colleges, Universities, and professional Schools That offer Various Faculty Development Services

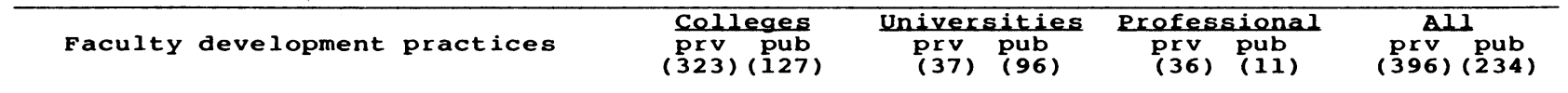

\section{Workshops or seminars on}

1) various methods or techniques of instruction

\begin{tabular}{|c|c|c|c|c|c|c|c|}
\hline 61 & 61 & 62 & 73 & 47 & 64 & 60 & 66 \\
\hline 38 & 24 & 30 & 41 & 39 & 55 & 38 & 32 \\
\hline 32 & 29 & 27 & 52 & 50 & 64 & 33 & 40 \\
\hline 62 & 57 & 57 & 42 & 33 & 27 & 59 & 50 \\
\hline 16 & 34 & 32 & 45 & 33 & 45 & 19 & 39 \\
\hline 19 & 28 & 27 & 35 & 13 & 18 & 19 & 31 \\
\hline 39 & 40 & 27 & 40 & 31 & 9 & 37 & 38 \\
\hline 26 & 29 & 16 & 40 & 31 & 18 & 25 & 33 \\
\hline 40 & 31 & 22 & 46 & 11 & 27 & 35 & 37 \\
\hline 29 & 28 & 22 & 46 & 25 & 45 & 28 & 36 \\
\hline
\end{tabular}

2) course or curricular planning

3) testing and evaluating student performance

4) academic advising and counseling skilis

5) research and scholarship skills

6) improving the management of departmental operations

7) general issues or trends in higher education (e.g.., competency testing. general education

8) personal development (e.g.. improving interpersonal skilis, values clarification, career planning)

9) understanding college students (e.g.. learning styles, developmental patterns)

10) theories and principles of

instruction

prv pub prv pub

(2)


TABLE 2-Continued

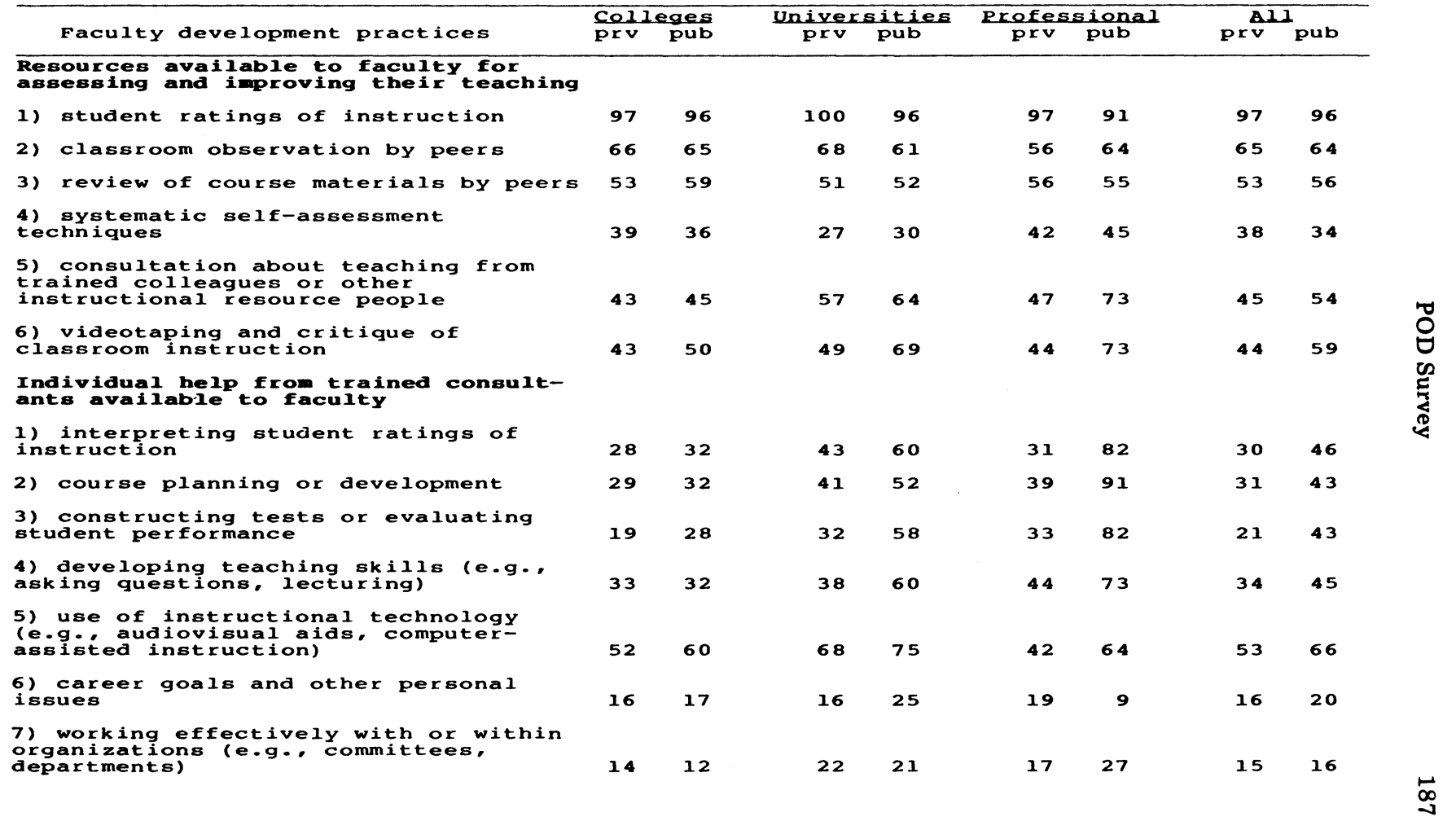


TABLE 2-Continued

\begin{tabular}{l} 
Faculty development practices \\
\hline Grants or awards. leaves. exchanges \\
1) grants for faculty members devel- \\
oping new or different approaches
\end{tabular}
oping new or different approaches to courses or teaching

colleges

prv pub

Universities

prv pub

Professional

prv pub

All

prv pub

2) faculty exchange program with other institutions

3) sabbatical leaves with at least half salary

$\begin{array}{llllllll}64 & 74 & 49 & 72 & 33 & 36 & 60 & 71 \\ 29 & 61 & 43 & 58 & 28 & 27 & 30 & 58 \\ 78 & 83 & 78 & 83 & 78 & 73 & 78 & 83 \\ 68 & 78 & 62 & 71 & 36 & 64 & 65 & 74 \\ 19 & 17 & 27 & 30 & 39 & 18 & 21 & 23 \\ 54 & 69 & 70 & 70 & 44 & 36 & 55 & 68 \\ 59 & 65 & 62 & 65 & 42 & 27 & 58 & 63 \\ 93 & 94 & 92 & 94 & 83 & 82 & 92 & 93 \\ 64 & 59 & 68 & 63 & 31 & 18 & 61 & 59\end{array}$

4) a policy of unpaid leaves covering educational or development purposes

5) a lighter than normal teaching load for first year faculty

6) temporary teaching load reductions to work on new course, major course revision, or research area

7) travel grants to refresh or update knowledge in a particular field

8) travel funds available for attending professional conferences

$64 \quad 59$

$31 \quad 18$

$61 \quad 59$ 
TABLE 2-Continued

\begin{tabular}{|c|c|c|c|c|c|c|c|c|}
\hline \multirow{2}{*}{ Faculty development practices } & \multicolumn{2}{|c|}{ colleges } & \multicolumn{2}{|c|}{ Universities } & \multicolumn{2}{|c|}{ Professional } & \multicolumn{2}{|c|}{ A11 } \\
\hline & prv & pub & prv & pub & prv & pub & prv & pub \\
\hline \multicolumn{9}{|l|}{ Other practices } \\
\hline $\begin{array}{l}\text { 1) special professional library } \\
\text { readily accessible to faculty con- } \\
\text { cerned with instructional method- } \\
\text { ology, teaching skilis, psychology } \\
\text { of learning, and similar topics }\end{array}$ & 33 & 40 & 38 & 48 & 44 & 73 & 35 & 45 \\
\hline $\begin{array}{l}\text { 2) annual awards to faculty for } \\
\text { excellence in teaching }\end{array}$ & 50 & 72 & 81 & 91 & 44 & 100 & 53 & 81 \\
\hline $\begin{array}{l}\text { 3) professional and personal develop- } \\
\text { ment plan (often called "growth } \\
\text { contracts") for individual faculty } \\
\text { members }\end{array}$ & 29 & 24 & 22 & 23 & 17 & 18 & 28 & 23 \\
\hline $\begin{array}{l}\text { 4) circulation of newsletter, arti- } \\
\text { cles etc. that are pertinent to teach- } \\
\text { ing improvement or faculty development }\end{array}$ & 49 & 36 & 32 & 56 & 42 & 45 & 47 & 45 \\
\hline $\begin{array}{l}\text { 5) a specific calendar period set } \\
\text { aside for professional development }\end{array}$ & 26 & 19 & 14 & 11 & 25 & $\mathbf{0}$ & 24 & 15 \\
\hline $\begin{array}{l}\text { 6) a visiting scholars program that } \\
\text { brings people to the campus for } \\
\text { short or long periods of time }\end{array}$ & 46 & 57 & 51 & 73 & 58 & 55 & 47 & 63 \\
\hline $\begin{array}{l}\text { 7) a periodic review of all faculty } \\
\text { members, whether tenured or not }\end{array}$ & 76 & 80 & 68 & 77 & 50 & 55 & 72 & 78 \\
\hline $\begin{array}{l}\text { 8) a campus committee on faculty } \\
\text { development }\end{array}$ & 67 & 61 & 51 & 58 & 53 & 9 & 64 & 58 \\
\hline
\end{tabular}


TABLE 3

Summary by Region. Institutional Type, and Enrollment of the Median Number of lo Faculty

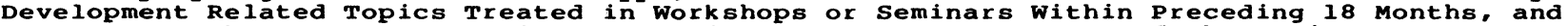
the Number of Institutions Which Treated Half or More of the Topics

\begin{tabular}{|c|c|c|c|c|c|c|c|c|c|c|c|c|c|c|c|}
\hline $\begin{array}{l}\text { Region and } \\
\text { institutional } \\
\text { type }\end{array}$ & $\mathbf{N}$ & $\begin{array}{l}\text { Under } \\
2,500 \\
\text { Mdn }\end{array}$ & $>4$ & $\mathbf{N}$ & $\begin{array}{l}2.500 \\
5.000 \\
\text { Man }\end{array}$ & $>4$ & $\mathbf{N}$ & $\begin{array}{l}5.000 \\
10.00 \\
M a n\end{array}$ & $>4$ & $\mathbf{N}$ & $\begin{array}{l}\text { Over } \\
10.00 \\
\text { Mdn }\end{array}$ & $>4$ & $\mathbf{N}$ & $\begin{array}{l}\text { All } \\
\text { Mdn }\end{array}$ & $>4$ \\
\hline $\begin{array}{l}\text { Northeast } \\
\text { Four-year } \\
\text { University } \\
\text { Professional }\end{array}$ & $\begin{array}{r}82 \\
3 \\
11\end{array}$ & $\begin{array}{l}3 \cdot 0 \\
2.0 \\
1.0\end{array}$ & $\begin{array}{r}20 \\
0 \\
2\end{array}$ & $\begin{array}{r}20 \\
4 \\
0\end{array}$ & $\begin{array}{l}3 \cdot 0 \\
2.0 \\
-\end{array}$ & $\begin{array}{l}4 \\
0 \\
0\end{array}$ & $\begin{array}{r}11 \\
7 \\
0\end{array}$ & $\begin{array}{l}3.0 \\
3.0 \\
-\end{array}$ & $\begin{array}{l}\mathbf{3} \\
\mathbf{0} \\
\mathbf{0}\end{array}$ & $\begin{array}{r}6 \\
13 \\
0\end{array}$ & $\begin{array}{c}4 \cdot 5 \\
6 \cdot 0 \\
-\end{array}$ & $\begin{array}{l}3 \\
\mathbf{8} \\
\mathbf{0}\end{array}$ & $\begin{array}{r}119 \\
27 \\
11\end{array}$ & $\begin{array}{l}3 \cdot 0 \\
3 \cdot 0 \\
1.0\end{array}$ & $\begin{array}{r}30 \\
8 \\
2\end{array}$ \\
\hline a $1 \perp$ NE & 96 & 3.0 & 22 & 24. & 3.0 & 4 & $1 \dot{8}$ & 3.0 & 3 & 19 & 5.0 & 11 & 157 & 3.0 & 40 \\
\hline $\begin{array}{l}\text { Southeast } \\
\text { Four-year } \\
\text { University } \\
\text { Professional }\end{array}$ & $\begin{array}{r}81 \\
10\end{array}$ & $\frac{3.0}{3.5}$ & $\begin{array}{r}20 \\
1 \\
2\end{array}$ & $\begin{array}{r}17 \\
5 \\
1\end{array}$ & $\begin{array}{l}2.0 \\
1.0 \\
-\end{array}$ & $\begin{array}{l}4 \\
1 \\
1\end{array}$ & $\begin{array}{l}9 \\
6 \\
0\end{array}$ & $\begin{array}{c}4.0 \\
5.5 \\
-\end{array}$ & $\begin{array}{l}3 \\
4 \\
0\end{array}$ & $\begin{array}{r}5 \\
18 \\
1\end{array}$ & $\begin{array}{c}3.0 \\
5.0 \\
-\end{array}$ & $\begin{array}{r}1 \\
11 \\
0\end{array}$ & $\begin{array}{r}112 \\
30 \\
12\end{array}$ & $\begin{array}{l}3.0 \\
5.0 \\
3.5\end{array}$ & $\begin{array}{r}28 \\
17 \\
3\end{array}$ \\
\hline a11 SE & 92 & 3.0 & 23 & 23 & 2.0 & 6 & 15 & 4.0 & 7 & 24 & 4.5 & 12 & 154 & 3.0 & 48 \\
\hline $\begin{array}{l}\text { Midwest } \\
\text { Four-year } \\
\text { University } \\
\text { Professional }\end{array}$ & $\begin{array}{r}120 \\
3 \\
15\end{array}$ & $\begin{array}{l}4 \cdot 0 \\
2.0 \\
3.5\end{array}$ & $\begin{array}{r}48 \\
1 \\
5\end{array}$ & $\begin{array}{r}17 \\
1 \\
0\end{array}$ & $\begin{array}{l}4.0 \\
-\end{array}$ & $\begin{array}{l}5 \\
0 \\
0\end{array}$ & $\begin{array}{r}11 \\
9 \\
0\end{array}$ & $\begin{array}{l}6.0 \\
5.0 \\
-\end{array}$ & $\begin{array}{l}6 \\
4 \\
0\end{array}$ & $\begin{array}{r}5 \\
24 \\
0\end{array}$ & $\begin{array}{l}5.0 \\
5.0 \\
-\end{array}$ & $\begin{array}{r}3 \\
12 \\
0\end{array}$ & $\begin{array}{r}153 \\
37 \\
15\end{array}$ & $\begin{array}{l}4.0 \\
5.0 \\
3.5\end{array}$ & $\begin{array}{r}62 \\
17 \\
5\end{array}$ \\
\hline a11 MW & 138 & 4.0 & 54 & 18 & 4.0 & 5 & 20 & 5.5 & 10 & 29 & 5.0 & 15 & 205 & 4.0 & 84 \\
\hline $\begin{array}{l}\text { West } \\
\text { Four-year } \\
\text { University } \\
\text { Professional }\end{array}$ & $\begin{array}{r}43 \\
3 \\
8\end{array}$ & $\begin{array}{l}4.0 \\
4.0 \\
2.5\end{array}$ & $\begin{array}{r}16 \\
1 \\
2\end{array}$ & $\begin{array}{r}11 \\
4 \\
0\end{array}$ & $\begin{array}{c}5.0 \\
2.0 \\
-\end{array}$ & $\begin{array}{l}\mathbf{6} \\
\mathbf{0} \\
0\end{array}$ & $\begin{array}{l}5 \\
7 \\
1\end{array}$ & $\begin{array}{c}5.0 \\
4.5 \\
-\end{array}$ & $\begin{array}{l}3 \\
3 \\
0\end{array}$ & $\begin{array}{r}7 \\
25 \\
0\end{array}$ & $\begin{array}{c}5.0 \\
5.0 \\
=\end{array}$ & $\begin{array}{r}4 \\
17 \\
0\end{array}$ & $\begin{array}{r}66 \\
39 \\
9\end{array}$ & $\begin{array}{l}4.0 \\
5.0 \\
2.0\end{array}$ & $\begin{array}{l}29 \\
21 \\
2\end{array}$ \\
\hline a11 w & 54 & 4.0 & 19 & 15 & $3 \cdot 5$ & 6 & 13 & 4.5 & 6 & 32 & 5.0 & 21 & 114 & 4.0 & 52 \\
\hline $\begin{array}{l}\text { A11 } \\
\text { Four-year } \\
\text { University } \\
\text { Professional }\end{array}$ & $\begin{array}{r}326 \\
10 \\
44\end{array}$ & $\begin{array}{l}4 \cdot 0 \\
3 \cdot 0 \\
3 \cdot 0\end{array}$ & $\begin{array}{r}104 \\
3 \\
11\end{array}$ & $\begin{array}{r}65 \\
14 \\
1\end{array}$ & $\begin{array}{l}3.0 \\
2.0 \\
-\end{array}$ & $\begin{array}{r}19 \\
1 \\
1\end{array}$ & $\begin{array}{r}36 \\
29 \\
1\end{array}$ & $\begin{array}{l}4.0 \\
4.0 \\
-\end{array}$ & $\begin{array}{r}15 \\
11 \\
0\end{array}$ & $\begin{array}{r}23 \\
80 \\
1\end{array}$ & $\begin{array}{l}4 \cdot 5 \\
5.0 \\
-\end{array}$ & $\begin{array}{r}11 \\
48 \\
0\end{array}$ & $\begin{array}{r}450 \\
133 \\
47\end{array}$ & $\begin{array}{l}4.0 \\
5.0 \\
3.0\end{array}$ & $\begin{array}{r}149 \\
63 \\
12\end{array}$ \\
\hline combined & 380 & 4.0 & 118 & 80 & 3.0 & 21 & 66 & 4.0 & 26 & 104 & 5.0 & 59 & 630 & 4.0 & 224 \\
\hline
\end{tabular}


TABLE 4

Summary by Region. Institutional Type, and Enrollment of the Median Number of six Teach-

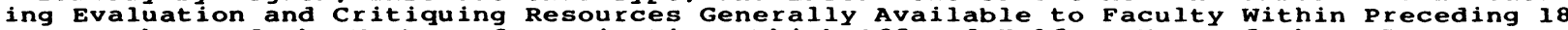
Months, and the Number of Institutions Which offered Half or More of Those Resources

\begin{tabular}{|c|c|c|c|c|c|c|c|c|c|c|c|c|c|c|c|}
\hline $\begin{array}{c}\text { Region and } \\
\text { institutional } \\
\text { type }\end{array}$ & $\mathbf{N}$ & $\begin{array}{l}\text { Under } \\
2.500 \\
\text { Mdn }\end{array}$ & $>3$ & $\mathbf{N}$ & $\begin{array}{l}2.500 \\
5.000 \\
\text { Man }\end{array}$ & $>3$ & $\mathbf{N}$ & $\begin{array}{l}5,000 \\
10,00 \\
\text { Man }\end{array}$ & $\overline{0}>3$ & $\mathbf{N}^{1}$ & $\begin{array}{l}\text { Over } \\
\text { o, oo o } \\
\text { Man }\end{array}$ & $>3$ & $\mathbf{N}$ & $\begin{array}{l}\text { All } \\
\text { Mdn }\end{array}$ & $>3$ \\
\hline $\begin{array}{l}\text { Northeast } \\
\text { Four-year } \\
\text { University } \\
\text { Professional }\end{array}$ & $\begin{array}{r}82 \\
3 \\
11\end{array}$ & $\begin{array}{l}3.0 \\
3.0 \\
3.0\end{array}$ & $\begin{array}{r}34 \\
1 \\
3\end{array}$ & $\begin{array}{r}20 \\
4 \\
0\end{array}$ & $\begin{array}{l}4 \cdot 0 \\
3.5 \\
-\end{array}$ & $\begin{array}{r}11 \\
2 \\
0\end{array}$ & $\begin{array}{r}11 \\
7 \\
0\end{array}$ & $\begin{array}{l}4.0 \\
4.0 \\
-\end{array}$ & $\begin{array}{l}8 \\
4 \\
0\end{array}$ & $\begin{array}{r}6 \\
13 \\
0\end{array}$ & $\begin{array}{l}4.5 \\
4.0 \\
-\end{array}$ & $\begin{array}{l}5 \\
7 \\
0\end{array}$ & $\begin{array}{r}119 \\
27 \\
11\end{array}$ & $\begin{array}{l}3.0 \\
4.0 \\
3.0\end{array}$ & $\begin{array}{r}58 \\
14 \\
3\end{array}$ \\
\hline a 11 NE & 96 & 3.0 & 38 & 24 & 4.0 & 13 & 18 & 4.0 & 12 & 19 & 4.0 & 12 & 157 & 3.0 & 75 \\
\hline $\begin{array}{l}\text { Southeast } \\
\text { Four-year } \\
\text { University } \\
\text { Professional }\end{array}$ & $\begin{array}{r}81 \\
11 \\
10\end{array}$ & $\begin{array}{l}3.0 \\
3.0\end{array}$ & $\begin{array}{r}27 \\
1 \\
4\end{array}$ & $\begin{array}{r}17 \\
5 \\
1\end{array}$ & $\begin{array}{l}3.0 \\
2.0 \\
-\end{array}$ & $\begin{array}{l}\mathbf{5} \\
\mathbf{0} \\
\mathbf{1}\end{array}$ & $\begin{array}{l}9 \\
6 \\
0\end{array}$ & $\begin{array}{c}2.0 \\
3.0 \\
-\end{array}$ & $\begin{array}{l}1 \\
3 \\
0\end{array}$ & $\begin{array}{r}5 \\
18 \\
1\end{array}$ & $\begin{array}{l}4.0 \\
4.0 \\
-\end{array}$ & $\begin{array}{r}3 \\
10 \\
1\end{array}$ & $\begin{array}{r}112 \\
30 \\
12\end{array}$ & $\begin{array}{l}3 \cdot 0 \\
3 \cdot 0 \\
3 \cdot 5\end{array}$ & $\begin{array}{r}36 \\
14 \\
6\end{array}$ \\
\hline al1 SE & 92 & 3.0 & 32 & 23 & 3.0 & 6 & 15 & 2.0 & 4 & 24 & 4.0 & 14 & 154 & 3.0 & 56 \\
\hline $\begin{array}{l}\text { Hidwest } \\
\text { Four-year } \\
\text { University } \\
\text { Professional }\end{array}$ & $\begin{array}{r}120 \\
15\end{array}$ & $\begin{array}{l}4.0 \\
3.5 \\
4.0\end{array}$ & $\begin{array}{r}67 \\
1 \\
9\end{array}$ & $\begin{array}{r}17 \\
1 \\
0\end{array}$ & $\begin{array}{c}3.0 \\
-\end{array}$ & $\begin{array}{l}7 \\
1 \\
0\end{array}$ & $\begin{array}{r}11 \\
9 \\
0\end{array}$ & $\begin{array}{l}5.0 \\
3.0 \\
-\end{array}$ & $\begin{array}{l}\mathbf{8} \\
\mathbf{3} \\
\mathbf{0}\end{array}$ & $\begin{array}{r}5 \\
24 \\
0\end{array}$ & $\begin{array}{l}3.0 \\
4.0 \\
-\end{array}$ & $\begin{array}{r}2 \\
13 \\
0\end{array}$ & $\begin{array}{r}153 \\
37 \\
15\end{array}$ & $\begin{array}{l}4 \cdot 0 \\
3 \cdot 5 \\
4 \cdot 0\end{array}$ & $\begin{array}{r}84 \\
18 \\
9\end{array}$ \\
\hline a11 MW & 138 & 4.0 & 77 & 18 & 3.0 & 8 & 20 & 4.0 & 11 & 29 & 4.0 & 15 & 205 & 4.0 & 111 \\
\hline $\begin{array}{l}\text { West } \\
\text { Four-year } \\
\text { University } \\
\text { Professional }\end{array}$ & $\begin{array}{r}43 \\
3 \\
8\end{array}$ & $\begin{array}{l}3.0 \\
2.0 \\
3.0\end{array}$ & $\begin{array}{r}19 \\
1 \\
2\end{array}$ & $\begin{array}{r}11 \\
4 \\
0\end{array}$ & $\begin{array}{c}3 \cdot 0 \\
2.5 \\
-\end{array}$ & $\begin{array}{l}\mathbf{5} \\
\mathbf{0} \\
\mathbf{0}\end{array}$ & $\begin{array}{l}\mathbf{5} \\
\mathbf{7} \\
\mathbf{1}\end{array}$ & $\begin{array}{c}4.0 \\
3.0 \\
-\end{array}$ & $\begin{array}{l}4 \\
3 \\
0\end{array}$ & $\begin{array}{r}7 \\
25 \\
0\end{array}$ & $\begin{array}{l}4.0 \\
4.0 \\
-\end{array}$ & $\begin{array}{r}4 \\
16 \\
0\end{array}$ & $\begin{array}{r}66 \\
39 \\
9\end{array}$ & $\begin{array}{l}3 \cdot 0 \\
4 \cdot 0 \\
3 \cdot 0\end{array}$ & $\begin{array}{r}32 \\
20 \\
2\end{array}$ \\
\hline $\operatorname{a11} w$ & 54 & 3.0 & 22 & 15 & 3.0 & 5 & 13 & 4.0 & 7 & 32 & 4.0 & 20 & 114 & 3.0 & 54 \\
\hline $\begin{array}{l}\text { A11 } \\
\text { Four-year } \\
\text { University } \\
\text { Professional }\end{array}$ & $\begin{array}{r}326 \\
10 \\
44\end{array}$ & $\begin{array}{l}3 \cdot \mathbf{0} \\
3 \cdot \mathbf{0} \\
\mathbf{3} \cdot \mathbf{0}\end{array}$ & $\begin{array}{r}147 \\
4 \\
18\end{array}$ & $\begin{array}{r}65 \\
14 \\
1\end{array}$ & $\begin{array}{l}3.0 \\
3.0 \\
-\end{array}$ & $\begin{array}{r}28 \\
3 \\
1\end{array}$ & $\begin{array}{r}36 \\
29 \\
1\end{array}$ & $\begin{array}{l}4.0 \\
3.0 \\
-\end{array}$ & $\begin{array}{r}21 \\
13 \\
0\end{array}$ & $\begin{array}{r}23 \\
80 \\
1\end{array}$ & $\begin{array}{c}4.0 \\
4.0 \\
-\end{array}$ & $\begin{array}{r}14 \\
46 \\
1\end{array}$ & $\begin{array}{r}450 \\
133 \\
47\end{array}$ & $\begin{array}{l}3 \cdot 0 \\
3 \cdot 5 \\
3.0\end{array}$ & $\begin{array}{r}210 \\
66 \\
20\end{array}$ \\
\hline combined & 380 & 3.0 & 169 & 80 & 3.0 & 32 & 66 & 4.0 & 34 & 104 & 4.0 & 61 & 630 & 3.0 & 296 \\
\hline
\end{tabular}




\section{TABLE 5}

Summary by Region. Institutional Type, and Enrollment of the Median Number of seven

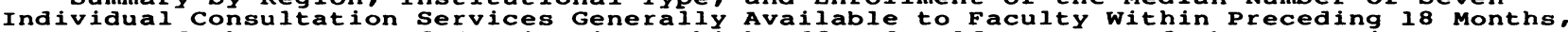
and the Number of Institutions Which offered Half or More of Those Services

\begin{tabular}{|c|c|c|c|c|c|c|c|c|c|c|c|c|c|c|c|}
\hline $\begin{array}{c}\text { Region and } \\
\text { institutional } \\
\text { type }\end{array}$ & $\mathbf{N}$ & $\begin{array}{l}\text { Under } \\
2.500 \\
\text { Mdn }\end{array}$ & $>3$ & $\mathbf{N}$ & $\begin{array}{l}2.500 \\
5.000 \\
\text { Man }\end{array}$ & $>3$ & $\mathbf{N}$ & $\begin{array}{l}5.000 \\
10.00 \\
\text { Man }\end{array}$ & $>3$ & $\mathbf{N}$ & $\begin{array}{l}\text { Over } \\
\text { 1.00 } \\
\text { Man }\end{array}$ & $>3$ & $\mathbf{N}$ & $\begin{array}{l}\text { All } \\
\text { Mdn }\end{array}$ & $>3$ \\
\hline $\begin{array}{l}\text { Northeast } \\
\text { Four-year } \\
\text { University } \\
\text { Professional }\end{array}$ & $\begin{array}{r}82 \\
3 \\
11\end{array}$ & $\begin{array}{l}1 \cdot 0 \\
0: 0 \\
2: 0\end{array}$ & $\begin{array}{l}9 \\
0 \\
3\end{array}$ & $\begin{array}{r}20 \\
4 \\
0\end{array}$ & $\begin{array}{c}1 \cdot 0 \\
1.0 \\
-\end{array}$ & $\begin{array}{l}\mathbf{5} \\
\mathbf{0} \\
\mathbf{0}\end{array}$ & $\begin{array}{r}11 \\
7 \\
0\end{array}$ & $\begin{array}{l}1.0 \\
1.0 \\
-\end{array}$ & $\begin{array}{l}3 \\
\mathbf{2} \\
\mathbf{0}\end{array}$ & $\begin{array}{r}6 \\
13 \\
0\end{array}$ & $\begin{array}{l}3.0 \\
5.0 \\
-\end{array}$ & $\begin{array}{r}2 \\
10 \\
0\end{array}$ & $\begin{array}{r}119 \\
27 \\
11\end{array}$ & $\begin{array}{l}1.0 \\
2.0 \\
2.0\end{array}$ & $\begin{array}{r}19 \\
12 \\
3\end{array}$ \\
\hline a 11 NE & 96 & 1.0 & 12 & 24 & 1.0 & 5 & 18 & 1.0 & 5 & 19 & 5.0 & 12 & 157 & 1.0 & 34 \\
\hline $\begin{array}{l}\text { Southeast } \\
\text { Four-year } \\
\text { University } \\
\text { Professional }\end{array}$ & $\begin{array}{r}81 \\
10\end{array}$ & $\begin{array}{l}1.0 \\
4.5\end{array}$ & $\begin{array}{r}10 \\
1 \\
7\end{array}$ & $\begin{array}{r}17 \\
5 \\
1\end{array}$ & $\begin{array}{c}2.0 \\
0.0 \\
-\end{array}$ & $\begin{array}{l}4 \\
1 \\
1\end{array}$ & $\begin{array}{l}9 \\
6 \\
0\end{array}$ & $\begin{array}{r}2.0 \\
5.0 \\
=\end{array}$ & $\begin{array}{l}2 \\
3 \\
0\end{array}$ & $\begin{array}{r}5 \\
18 \\
1\end{array}$ & $\begin{array}{c}5.0 \\
4.0 \\
-\end{array}$ & $\begin{array}{r}3 \\
11 \\
1\end{array}$ & $\begin{array}{r}112 \\
30 \\
12\end{array}$ & $\begin{array}{l}1.0 \\
4.0 \\
5.0\end{array}$ & $\begin{array}{r}19 \\
16 \\
9\end{array}$ \\
\hline a 11 SE & 92 & 1.0 & 18 & 23 & 2.0 & 6 & 15 & 2.0 & 5 & 24 & 4.0 & 15 & 154 & 2.0 & 44 \\
\hline $\begin{array}{l}\text { Midwest } \\
\text { Four-year } \\
\text { University } \\
\text { Professional }\end{array}$ & $\begin{array}{r}120 \\
3 \\
15\end{array}$ & $\begin{array}{l}3 \cdot 0 \\
1.5 \\
5 \cdot 5\end{array}$ & $\begin{array}{r}32 \\
0 \\
5\end{array}$ & $\begin{array}{r}17 \\
1 \\
0\end{array}$ & $\stackrel{2.5}{-}$ & $\begin{array}{l}2 \\
1 \\
0\end{array}$ & $\begin{array}{r}11 \\
9 \\
0\end{array}$ & $\begin{array}{l}4 \cdot 0 \\
4.5 \\
-\end{array}$ & $\begin{array}{l}7 \\
4 \\
0\end{array}$ & $\begin{array}{r}5 \\
24 \\
0\end{array}$ & $\begin{array}{l}4.0 \\
5.0 \\
-\end{array}$ & $\begin{array}{r}2 \\
16 \\
0\end{array}$ & $\begin{array}{r}153 \\
37 \\
15\end{array}$ & $\begin{array}{l}3 \cdot 0 \\
5 \cdot 0 \\
5 \cdot 5\end{array}$ & $\begin{array}{r}43 \\
21 \\
5\end{array}$ \\
\hline $\mathbf{a} 11 \mathrm{MW}$ & 138 & 3.0 & 37 & 18 & 3.0 & 3 & 20 & 4.0 & 11 & 29 & 5.0 & 18 & 205 & 3.0 & 69 \\
\hline $\begin{array}{l}\text { west } \\
\text { Four-year } \\
\text { University } \\
\text { Professional }\end{array}$ & $\begin{array}{r}43 \\
3 \\
8\end{array}$ & $\begin{array}{l}2.0 \\
3.0 \\
2.5\end{array}$ & $\begin{array}{l}6 \\
0 \\
2\end{array}$ & $\begin{array}{r}11 \\
4 \\
0\end{array}$ & $\begin{array}{l}3 \cdot 5 \\
1.0 \\
-\end{array}$ & $\begin{array}{l}4 \\
0 \\
0\end{array}$ & $\begin{array}{l}5 \\
7 \\
1\end{array}$ & $\begin{array}{l}4.0 \\
3: 0 \\
-\end{array}$ & $\begin{array}{l}4 \\
1 \\
0\end{array}$ & $\begin{array}{r}7 \\
25 \\
0\end{array}$ & $\begin{array}{l}3 \cdot 5 \\
4.0 \\
-\end{array}$ & $\begin{array}{r}2 \\
17 \\
0\end{array}$ & $\begin{array}{r}66 \\
39 \\
9\end{array}$ & $\begin{array}{l}3 \cdot 0 \\
4.0 \\
2.5\end{array}$ & $\begin{array}{r}16 \\
18 \\
2\end{array}$ \\
\hline $\operatorname{a11} \mathrm{w}$ & 54 & 2.0 & 8 & 15 & 3.0 & 4 & 13 & 4.0 & 5 & 32 & 4.0 & 19 & 114 & 3.0 & 36 \\
\hline $\begin{array}{l}\text { A11 } \\
\text { Four-year } \\
\text { University } \\
\text { Professional }\end{array}$ & $\begin{array}{r}326 \\
10 \\
44\end{array}$ & $\begin{array}{l}2 \cdot 0 \\
1 \cdot 5 \\
3 \cdot 0\end{array}$ & $\begin{array}{r}57 \\
1 \\
17\end{array}$ & $\begin{array}{r}65 \\
14 \\
1\end{array}$ & $\begin{array}{l}2 \cdot 0 \\
1.0 \\
-\end{array}$ & $\begin{array}{r}15 \\
2 \\
1\end{array}$ & $\begin{array}{r}36 \\
29 \\
1\end{array}$ & $\begin{array}{c}3.0 \\
3.0 \\
=\end{array}$ & $\begin{array}{r}16 \\
10 \\
0\end{array}$ & $\begin{array}{r}23 \\
80 \\
1\end{array}$ & $\begin{array}{c}3.0 \\
5.0 \\
-\end{array}$ & $\begin{array}{r}9 \\
54 \\
1\end{array}$ & $\begin{array}{r}450 \\
133 \\
47\end{array}$ & $\begin{array}{l}2.0 \\
4.0 \\
4.0\end{array}$ & $\begin{array}{l}97 \\
67 \\
19\end{array}$ \\
\hline combined & 380 & 2.0 & 75 & 80 & 2.0 & 18 & 66 & 3.0 & 26 & 104 & 5.0 & 64 & 630 & 2.0 & 183 \\
\hline
\end{tabular}


TABLE 6

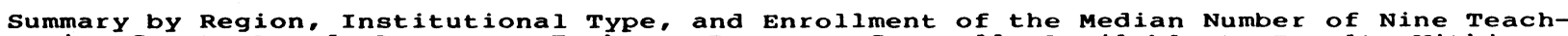
ing Grant. Award, Leave, or Exchange Programs Generally Available to Faculty Within

Preceding 18 Months, and the Number of Institutions offering Half or More of Them

\begin{tabular}{|c|c|c|c|c|c|c|c|c|c|c|c|c|c|c|c|}
\hline $\begin{array}{c}\text { Region and } \\
\text { institutional } \\
\text { type }\end{array}$ & $\mathbf{N}$ & $\begin{array}{l}\text { Under } \\
2,500 \\
\text { Man }\end{array}$ & $>4$ & $\mathbf{N}$ & $\begin{array}{l}2.500 \\
5.000 \\
\operatorname{Man}\end{array}$ & $>4$ & $\mathbf{N}$ & $\begin{array}{l}5.00 \\
10.00 \\
\text { Man }\end{array}$ & & $\mathbf{N}$ & $\begin{array}{l}\text { Over } \\
10.00 \\
\text { Man }\end{array}$ & $>4$ & $\mathbf{N}$ & $\begin{array}{l}\text { All } \\
\text { Mdn }\end{array}$ & $>4$ \\
\hline $\begin{array}{l}\text { Northeast } \\
\text { Four-year } \\
\text { University } \\
\text { Professional }\end{array}$ & $\begin{array}{r}82 \\
3 \\
11\end{array}$ & $\begin{array}{l}6 \cdot 0 \\
8.0 \\
3.0\end{array}$ & $\begin{array}{r}56 \\
3 \\
2\end{array}$ & $\begin{array}{r}20 \\
4 \\
0\end{array}$ & $\begin{array}{c}7.5 \\
5.5 \\
-\end{array}$ & $\begin{array}{r}17 \\
4 \\
0\end{array}$ & $\begin{array}{r}11 \\
0\end{array}$ & $\begin{array}{c}8.0 \\
4.0 \\
-\end{array}$ & $\begin{array}{r}10 \\
3 \\
0\end{array}$ & $\begin{array}{r}6 \\
13 \\
0\end{array}$ & $\begin{array}{c}7.5 \\
7: 0 \\
-\end{array}$ & $\begin{array}{r}6 \\
11 \\
0\end{array}$ & $\begin{array}{r}119 \\
27 \\
11\end{array}$ & $\begin{array}{l}6.0 \\
6.0 \\
3.0\end{array}$ & $\begin{array}{r}89 \\
21 \\
2\end{array}$ \\
\hline a 11 NE & 96 & 5.0 & 61 & 24 & 7.0 & 21 & 18 & 6.0 & 13 & 19 & 7.0 & 17 & 157 & 6.0 & 112 \\
\hline $\begin{array}{l}\text { Southeast } \\
\text { Four-year } \\
\text { University } \\
\text { Professional }\end{array}$ & $\begin{array}{r}81 \\
10\end{array}$ & $\begin{array}{l}5.0 \\
4.0\end{array}$ & $\begin{array}{r}55 \\
0 \\
5\end{array}$ & $\begin{array}{r}17 \\
5 \\
1\end{array}$ & $\begin{array}{l}6.0 \\
5.0 \\
-\end{array}$ & $\begin{array}{r}11 \\
3 \\
1\end{array}$ & $\begin{array}{l}9 \\
6 \\
0\end{array}$ & $\begin{array}{c}4.0 \\
5 \div 5 \\
-\end{array}$ & $\begin{array}{l}4 \\
4 \\
0\end{array}$ & $\begin{array}{r}5 \\
18 \\
1\end{array}$ & $\begin{array}{c}7 \cdot 0 \\
7 \cdot 0 \\
-\end{array}$ & $\begin{array}{r}4 \\
15 \\
1\end{array}$ & $\begin{array}{r}112 \\
30 \\
12\end{array}$ & $\begin{array}{l}5.0 \\
6: 0 \\
5.0\end{array}$ & $\begin{array}{r}74 \\
22 \\
7\end{array}$ \\
\hline al1 SE & 92 & 5.0 & 60 & 23 & 6.0 & 15 & 15 & 5.0 & 8 & 24 & 7.0 & 20 & 154 & 5.0 & 103 \\
\hline $\begin{array}{l}\text { Midwest } \\
\text { Four-year } \\
\text { University } \\
\text { Professional }\end{array}$ & $\begin{array}{r}120 \\
3 \\
15\end{array}$ & $\begin{array}{l}6.0 \\
3.0 \\
4.0\end{array}$ & $\begin{array}{r}82 \\
1 \\
6\end{array}$ & $\begin{array}{r}17 \\
1 \\
0\end{array}$ & $\begin{array}{c}6.0 \\
=\end{array}$ & $\begin{array}{r}15 \\
\mathbf{0} \\
0\end{array}$ & $\begin{array}{r}11 \\
9 \\
0\end{array}$ & $\begin{array}{c}6.0 \\
7.0 \\
-\end{array}$ & $\begin{array}{r}10 \\
8 \\
0\end{array}$ & $\begin{array}{r}5 \\
24 \\
0\end{array}$ & $\begin{array}{c}6 \cdot 0 \\
6.0 \\
-\end{array}$ & $2 \frac{4}{0}$ & $\begin{array}{r}153 \\
37 \\
15\end{array}$ & $\begin{array}{l}6 \cdot 0 \\
7.0 \\
4.0\end{array}$ & $\begin{array}{r}111 \\
30 \\
6\end{array}$ \\
\hline al1 MW & 138 & 6.0 & 89 & 18 & 6.0 & 15 & 20 & 7.0 & 18 & 29 & 6.0 & 25 & 205 & 6.0 & 147 \\
\hline $\begin{array}{l}\text { West } \\
\text { Four-year } \\
\text { University } \\
\text { Professional }\end{array}$ & $\begin{array}{r}43 \\
3 \\
8\end{array}$ & $\begin{array}{l}5.0 \\
4.0 \\
5.0\end{array}$ & $\begin{array}{r}27 \\
0 \\
5\end{array}$ & $\begin{array}{r}11 \\
4 \\
0\end{array}$ & $\begin{array}{c}7.0 \\
6: 5 \\
-\end{array}$ & $\begin{array}{l}8 \\
3 \\
0\end{array}$ & $\begin{array}{l}5 \\
7 \\
1\end{array}$ & $\begin{array}{c}7.0 \\
5.0 \\
-\end{array}$ & $\begin{array}{l}5 \\
4 \\
0\end{array}$ & $\begin{array}{r}7 \\
25 \\
0\end{array}$ & $\begin{array}{l}6.0 \\
7: 0 \\
-\end{array}$ & $\begin{array}{r}7 \\
19 \\
0\end{array}$ & $\begin{array}{r}66 \\
39 \\
9\end{array}$ & $\begin{array}{l}6.0 \\
6.0 \\
5.0\end{array}$ & $\begin{array}{r}47 \\
26 \\
5\end{array}$ \\
\hline a11 w & 54 & 5.0 & 32 & 15 & 7.0 & 11 & 13 & 5.0 & 9 & 32 & 7.0 & 26 & 114 & 6.0 & 78 \\
\hline $\begin{array}{l}\text { A11 } \\
\text { Four-year } \\
\text { University } \\
\text { Professional }\end{array}$ & $\begin{array}{r}326 \\
10 \\
44\end{array}$ & $\begin{array}{l}5.0 \\
4.0 \\
4.0\end{array}$ & $\begin{array}{r}220 \\
4 \\
18\end{array}$ & $\begin{array}{r}65 \\
14 \\
1\end{array}$ & $\begin{array}{c}6.0 \\
5.5 \\
-\end{array}$ & $\begin{array}{r}51 \\
10 \\
1\end{array}$ & $\begin{array}{r}36 \\
29 \\
1\end{array}$ & $\begin{array}{c}6.5 \\
5.0 \\
-\end{array}$ & $\begin{array}{r}29 \\
19 \\
0\end{array}$ & $\begin{array}{r}23 \\
80 \\
1\end{array}$ & $\begin{array}{c}6.0 \\
7: 0 \\
-\end{array}$ & $\begin{array}{r}21 \\
66 \\
1\end{array}$ & $\begin{array}{r}450 \\
133 \\
47\end{array}$ & $\begin{array}{l}6.0 \\
6.0 \\
4.0\end{array}$ & $\begin{array}{r}321 \\
99 \\
20\end{array}$ \\
\hline combined & 380 & 5.0 & 242 & 80 & 6.0 & 62 & 66 & 6.0 & 48 & 104 & 7.0 & 88 & 630 & 6.0 & 440 \\
\hline
\end{tabular}


TABLE 7

Survey by Region. Institutional Type, and Enrollment of the Median Number of Eight

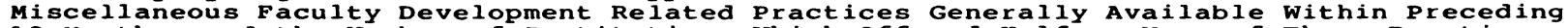
18 Months, and the Number of Institutions Which offered Half or More of Those Practices

\begin{tabular}{|c|c|c|c|c|c|c|c|c|c|c|c|c|c|c|c|}
\hline $\begin{array}{c}\text { Region and } \\
\text { institutional } \\
\text { type }\end{array}$ & $\mathbf{N}$ & $\begin{array}{l}\text { Under } \\
2.500 \\
\text { Man }\end{array}$ & $>4$ & $\mathbf{N}$ & $\begin{array}{l}2.500 \\
5: 000 \\
\operatorname{Man}\end{array}$ & $>4$ & $\mathbf{N}$ & $\begin{array}{l}5,000 \\
10,00 \\
\text { Man }\end{array}$ & $>4$ & $\mathbf{N}$ & $\begin{array}{l}\text { Over } \\
10.00 \\
\text { Man }\end{array}$ & $>4$ & $\mathbf{N}$ & $\begin{array}{l}\text { Al } 1 \\
\text { Man }\end{array}$ & $>4$ \\
\hline $\begin{array}{l}\text { Rortheast } \\
\text { Four-year } \\
\text { University } \\
\text { Professional }\end{array}$ & $\begin{array}{r}82 \\
3 \\
11\end{array}$ & $\begin{array}{l}3.0 \\
2.0 \\
2.0\end{array}$ & $\begin{array}{r}21 \\
1 \\
0\end{array}$ & $\begin{array}{r}20 \\
4 \\
0\end{array}$ & $\begin{array}{c}3.0 \\
2.5 \\
-\end{array}$ & $\begin{array}{l}5 \\
1 \\
0\end{array}$ & $\begin{array}{r}11 \\
7 \\
0\end{array}$ & $\begin{array}{c}5.0 \\
3.0 \\
-\end{array}$ & $\begin{array}{l}6 \\
1 \\
0\end{array}$ & $\begin{array}{r}6 \\
13 \\
0\end{array}$ & $\begin{array}{c}4.5 \\
4.0 \\
-\end{array}$ & $\begin{array}{l}3 \\
5 \\
0\end{array}$ & $\begin{array}{r}119 \\
27 \\
11\end{array}$ & $\begin{array}{l}4.0 \\
4.0 \\
2.0\end{array}$ & $\begin{array}{r}35 \\
8 \\
0\end{array}$ \\
\hline a 11 NE & 96 & 3.0 & 22 & 24 & 3.0 & 6 & 18. & 4.0 & 7 & 19 & 4.0 & 8 & 157 & 4.0 & 43 \\
\hline $\begin{array}{l}\text { Southeast } \\
\text { Four-year } \\
\text { University } \\
\text { Professional }\end{array}$ & $\begin{array}{r}81 \\
11 \\
10\end{array}$ & $\begin{array}{l}4.0 \\
3.5\end{array}$ & $\begin{array}{r}25 \\
0 \\
4\end{array}$ & $\begin{array}{r}17 \\
5 \\
1\end{array}$ & $\begin{array}{l}3 \cdot 0 \\
2.0 \\
-\end{array}$ & $\begin{array}{l}3 \\
0 \\
1\end{array}$ & $\begin{array}{l}9 \\
6 \\
0\end{array}$ & $\begin{array}{l}5.0 \\
5.0 \\
-\end{array}$ & $\begin{array}{l}5 \\
4 \\
0\end{array}$ & $\begin{array}{r}5 \\
18 \\
1\end{array}$ & $\begin{array}{l}4.0 \\
5.0 \\
-\end{array}$ & $\begin{array}{r}2 \\
11 \\
0\end{array}$ & $\begin{array}{r}112 \\
30 \\
12\end{array}$ & $\begin{array}{l}4.0 \\
4.5 \\
4.0\end{array}$ & $\begin{array}{r}35 \\
15 \\
5\end{array}$ \\
\hline aI1 SE & 92 & 4.0 & 29 & 23 & 3.0 & 4 & 15 & 5.0 & 9 & 24 & 5.0 & 13 & 154 & 4.0 & 55 \\
\hline $\begin{array}{l}\text { Hidwest } \\
\text { Four-year } \\
\text { University } \\
\text { Professional }\end{array}$ & $\begin{array}{r}120 \\
3 \\
15\end{array}$ & $\begin{array}{l}4.0 \\
2.0 \\
4.0\end{array}$ & $\begin{array}{r}50 \\
0 \\
6\end{array}$ & $\begin{array}{r}17 \\
1 \\
0\end{array}$ & $\begin{array}{c}4.0 \\
=\end{array}$ & $\begin{array}{l}\mathbf{6} \\
\mathbf{0} \\
\mathbf{0}\end{array}$ & $\begin{array}{r}11 \\
9 \\
0\end{array}$ & $\begin{array}{c}5.0 \\
5.0 \\
-\end{array}$ & $\begin{array}{l}7 \\
6 \\
0\end{array}$ & $\begin{array}{r}5 \\
24 \\
0\end{array}$ & $\begin{array}{c}3.0 \\
4.0 \\
-\end{array}$ & $\begin{array}{l}2 \\
\mathbf{8} \\
\mathbf{0}\end{array}$ & $\begin{array}{r}153 \\
37 \\
15\end{array}$ & $\begin{array}{l}4.0 \\
4.0 \\
4.0\end{array}$ & $\begin{array}{r}65 \\
14 \\
6\end{array}$ \\
\hline a11 MW & 138 & 4.0 & 56 & 18 & 4.0 & 6 & 20 & 5.0 & 13 & 29 & 4.0 & 10 & 205 & 4.0 & 85 \\
\hline $\begin{array}{l}\text { West } \\
\text { Four-year } \\
\text { University } \\
\text { Professional }\end{array}$ & $\begin{array}{r}43 \\
3 \\
8\end{array}$ & $\begin{array}{l}3.0 \\
2.0 \\
2.5\end{array}$ & $\begin{array}{r}11 \\
1 \\
1\end{array}$ & $\begin{array}{r}11 \\
4 \\
0\end{array}$ & $\begin{array}{c}5.0 \\
3.5 \\
-\end{array}$ & $\begin{array}{l}7 \\
0 \\
0\end{array}$ & $\begin{array}{l}5 \\
7 \\
1\end{array}$ & $\begin{array}{c}3.0 \\
4.0 \\
-\end{array}$ & $\begin{array}{l}0 \\
2 \\
0\end{array}$ & $\begin{array}{r}7 \\
25 \\
0\end{array}$ & $\begin{array}{c}4.0 \\
5.0 \\
-\end{array}$ & $\begin{array}{r}3 \\
16 \\
0\end{array}$ & $\begin{array}{r}66 \\
39 \\
9\end{array}$ & $\begin{array}{l}4.0 \\
4.5 \\
2.0\end{array}$ & $\begin{array}{r}21 \\
19 \\
1\end{array}$ \\
\hline $\operatorname{al1} w$ & 54 & 3.0 & 13 & 15 & 4.0 & 7 & 13 & $3 \cdot 5$ & 2 & 32 & 5.0 & 19 & 114 & 4.0 & 41 \\
\hline $\begin{array}{l}\text { A11 } \\
\text { Four-year } \\
\text { University } \\
\text { Professional }\end{array}$ & $\begin{array}{r}326 \\
10 \\
44\end{array}$ & $\begin{array}{l}4.0 \\
2.0 \\
3.0\end{array}$ & $\begin{array}{r}107 \\
2 \\
11\end{array}$ & $\begin{array}{r}65 \\
14 \\
1\end{array}$ & $\begin{array}{c}4.0 \\
2.0 \\
-\end{array}$ & $\begin{array}{r}21 \\
1 \\
1\end{array}$ & $\begin{array}{r}36 \\
29 \\
1\end{array}$ & $\begin{array}{r}4.5 \\
4.0 \\
-\end{array}$ & $\begin{array}{r}18 \\
13 \\
0\end{array}$ & $\begin{array}{r}23 \\
80 \\
1\end{array}$ & $\begin{array}{c}4.0 \\
5.0 \\
-\end{array}$ & $\begin{array}{r}10 \\
40 \\
0\end{array}$ & $\begin{array}{r}450 \\
133 \\
47\end{array}$ & $\begin{array}{l}4.0 \\
4.0 \\
3.0\end{array}$ & $\begin{array}{r}156 \\
56 \\
12\end{array}$ \\
\hline combined & 380 & 4.0 & 120 & 80 & 4.0 & 23 & 66 & 4.0 & 31 & 104 & 4.0 & 50 & 630 & 4.0 & 224 \\
\hline
\end{tabular}


TABLE 8

Summary by Region. Institutional Type, and Enrollment of the Median Number of 41 Faculty Development Programs and Activities Generally Available to Faculty within Preceding l8 Months. and the Number of Institutions Which offered Half or More of Those Services

\begin{tabular}{|c|c|c|c|c|c|c|c|c|c|c|c|c|c|c|c|}
\hline $\begin{array}{c}\text { Region and } \\
\text { institutional } \\
\text { type }\end{array}$ & $\mathbf{N}$ & $\begin{array}{l}\text { Under } \\
2,500 \\
\text { Man }\end{array}$ & $>20$ & $\mathbf{N}$ & $\begin{array}{l}2.500 \\
5.000 \\
\text { Man }\end{array}$ & $>20$ & $\mathbf{N}$ & $\begin{array}{l}5,000- \\
10,000 \\
\text { Man }\end{array}$ & $>20$ & $\mathbf{N}$ & $\begin{array}{l}\text { Over } \\
10,000 \\
\text { Man }\end{array}$ & $>20$ & $\mathbf{N}$ & $\begin{array}{l}\operatorname{Al} 1 \\
\operatorname{Mdn}\end{array}$ & $>20$ \\
\hline $\begin{array}{l}\text { Wortheast } \\
\text { Four-year } \\
\text { University } \\
\text { Professional }\end{array}$ & $\begin{array}{r}82 \\
3 \\
11\end{array}$ & $\begin{array}{l}16 \cdot 0 \\
17 \cdot 0 \\
13 \cdot 0\end{array}$ & $\begin{array}{r}18 \\
0 \\
1\end{array}$ & $\begin{array}{r}20 \\
4 \\
0\end{array}$ & $\begin{array}{l}19 \cdot 0 \\
15.5 \\
-\end{array}$ & $\begin{array}{l}7 \\
1 \\
0\end{array}$ & $\begin{array}{r}11 \\
7 \\
0\end{array}$ & $\begin{array}{c}19.0 \\
16.0 \\
-\end{array}$ & $\begin{array}{l}\mathbf{5} \\
\mathbf{1} \\
\mathbf{0}\end{array}$ & $\begin{array}{r}6 \\
13 \\
0\end{array}$ & $\begin{array}{c}24 \cdot 5 \\
24.0 \\
-\end{array}$ & $\begin{array}{r}4 \\
10\end{array}$ & $\begin{array}{r}119 \\
27 \\
11\end{array}$ & $\begin{array}{l}17 \cdot 0 \\
18 \cdot 0 \\
13 \cdot 0\end{array}$ & $\begin{array}{r}34 \\
12 \\
1\end{array}$ \\
\hline a11 NE & 96 & 16.0 & 19 & 24 & 18.5 & 8 & 18 & $18 \cdot 0$ & 6 & 19 & 24.0 & 14 & 157 & 17.0 & 47 \\
\hline $\begin{array}{l}\text { southeast } \\
\text { Four-year } \\
\text { University } \\
\text { Professional }\end{array}$ & $\begin{array}{r}81 \\
10\end{array}$ & $\begin{array}{l}17.0 \\
1 \overline{8} \cdot 0\end{array}$ & $\begin{array}{r}18 \\
1 \\
3\end{array}$ & $\begin{array}{r}17 \\
5 \\
1\end{array}$ & $\begin{array}{l}17 \cdot 0 \\
12 \cdot 0 \\
-\end{array}$ & $\begin{array}{l}4 \\
0 \\
1\end{array}$ & $\begin{array}{l}9 \\
6 \\
0\end{array}$ & $\begin{array}{c}16 \cdot 0 \\
25 \cdot 0 \\
-\end{array}$ & $\begin{array}{l}3 \\
4 \\
0\end{array}$ & $\begin{array}{r}5 \\
18 \\
1\end{array}$ & $\begin{array}{l}22.0 \\
23.0 \\
-\end{array}$ & $\begin{array}{r}3 \\
12 \\
1\end{array}$ & $\begin{array}{r}112 \\
30 \\
12\end{array}$ & $\begin{array}{l}17 \cdot 0 \\
22 \cdot 0 \\
19 \cdot 5\end{array}$ & $\begin{array}{r}28 \\
17 \\
5\end{array}$ \\
\hline all SE & 92 & 17.0 & 22 & 23 & 16.0 & 5 & 15 & 19.0 & 7 & 24 & 22.5 & 16 & 154 & 18.0 & 50 \\
\hline $\begin{array}{l}\text { Ridwest } \\
\text { Four-year } \\
\text { University } \\
\text { Professional }\end{array}$ & $\begin{array}{r}120 \\
3 \\
15\end{array}$ & $\begin{array}{l}19 \cdot 0 \\
13 \cdot 0 \\
16 \cdot 0\end{array}$ & $\begin{array}{r}54 \\
0 \\
7\end{array}$ & $\begin{array}{r}17 \\
1 \\
0\end{array}$ & $\begin{array}{c}19.0 \\
-\end{array}$ & $\begin{array}{l}\mathbf{7} \\
\mathbf{0} \\
\mathbf{0}\end{array}$ & $\begin{array}{r}11 \\
9 \\
0\end{array}$ & $\begin{array}{l}23 \cdot 0 \\
20 \cdot 0 \\
-\end{array}$ & $\begin{array}{l}9 \\
4 \\
0\end{array}$ & $\begin{array}{r}5 \\
24 \\
0\end{array}$ & $\begin{array}{l}18 \cdot 0 \\
21 \cdot 5 \\
-\end{array}$ & $\begin{array}{r}2 \\
13 \\
0\end{array}$ & $\begin{array}{r}153 \\
37 \\
15\end{array}$ & $\begin{array}{l}19 \cdot 0 \\
20 \cdot 0 \\
16 \cdot 0\end{array}$ & $\begin{array}{r}72 \\
17 \\
7\end{array}$ \\
\hline al1 MW & 138 & $18 \cdot 5$ & 61 & 18 & 18.0 & 7 & 20 & 23.0 & 13 & 29 & 21.0 & 15 & 205 & 19.0 & 96 \\
\hline $\begin{array}{l}\text { West } \\
\text { Four-year } \\
\text { University } \\
\text { Professional }\end{array}$ & $\begin{array}{r}43 \\
3 \\
8\end{array}$ & $\begin{array}{l}16 \cdot 0 \\
14.0 \\
15.0\end{array}$ & $\begin{array}{r}15 \\
1 \\
1\end{array}$ & $\begin{array}{r}11 \\
4 \\
0\end{array}$ & $\begin{array}{l}22 \cdot 0 \\
15.5 \\
-\end{array}$ & $\begin{array}{l}7 \\
0 \\
0\end{array}$ & $\begin{array}{l}5 \\
7 \\
1\end{array}$ & $\begin{array}{c}24.0 \\
18.0 \\
-\end{array}$ & $\begin{array}{l}4 \\
3 \\
0\end{array}$ & $\begin{array}{r}7 \\
25 \\
0\end{array}$ & $\begin{array}{l}21 \cdot 0 \\
25 \cdot 0 \\
-\end{array}$ & $\begin{array}{r}4 \\
20 \\
0\end{array}$ & $\begin{array}{r}66 \\
39 \\
9\end{array}$ & $\begin{array}{l}19 \cdot 0 \\
21 \cdot 0 \\
15 \cdot 0\end{array}$ & $\begin{array}{r}30 \\
24 \\
1\end{array}$ \\
\hline$a 11 w$ & 54 & 16.0 & 17 & 15 & 20.0 & 7 & 13 & 22.0 & 7 & 32 & 22.0 & 24 & 114 & 20.0 & 55 \\
\hline $\begin{array}{l}\text { A11 } \\
\text { Four-year } \\
\text { University } \\
\text { Professional }\end{array}$ & $\begin{array}{r}326 \\
10 \\
44\end{array}$ & $\begin{array}{l}17 \cdot 0 \\
15 \cdot 0 \\
15.0\end{array}$ & $\begin{array}{r}105 \\
2 \\
12\end{array}$ & $\begin{array}{r}65 \\
14 \\
1\end{array}$ & $\begin{array}{l}18.0 \\
15.0 \\
-\end{array}$ & $\begin{array}{r}25 \\
1 \\
1\end{array}$ & $\begin{array}{r}36 \\
29 \\
1\end{array}$ & $\begin{array}{l}21 \cdot 5 \\
18 \cdot 0 \\
-\end{array}$ & $\begin{array}{r}21 \\
12 \\
0\end{array}$ & $\begin{array}{r}23 \\
80 \\
1\end{array}$ & $\begin{array}{l}22.0 \\
23.0 \\
-\end{array}$ & $\begin{array}{r}13 \\
55 \\
1\end{array}$ & $\begin{array}{r}450 \\
133 \\
47\end{array}$ & $\begin{array}{l}18 \cdot 0 \\
21 \cdot 0 \\
15 \cdot 0\end{array}$ & $\begin{array}{r}164 \\
70 \\
14\end{array}$ \\
\hline combined & 380 & 17.0 & 119 & 80 & 17.0 & 27 & 66 & 20.5 & 33 & 104 & 23.0 & 69 & 630 & 18.0 & 248 \\
\hline
\end{tabular}


What we have, then, is evidence that the faculty development movement has survived the budget cuts and shifting interest of recent years, even if many specific programs have not. Probably half or more of our four-year colleges, universities, and professional schools offer some formal faculty development, instructional development, and/or teaching improvement services. Those activities are most often coordinated by a committee, an administrator whose primary responsibilities lie elsewhere, or a committee and administrator working together. We don't know many details (e.g., Are workshops one hour, one day, one week? Are consultations "one shot" or "longterm"?) Nor do we know who participates, what really works, what's just window dressing. However, we do have an extensive mailing list of those responsible for faculty development activities, and it is available to those who might seek answers to such questions.

\section{REFERENCES}

Centra, J. (1976). Faculty development practices in U.S. colleges and universities (Project Report 76-30). Princeton: Educational Testing Service. 